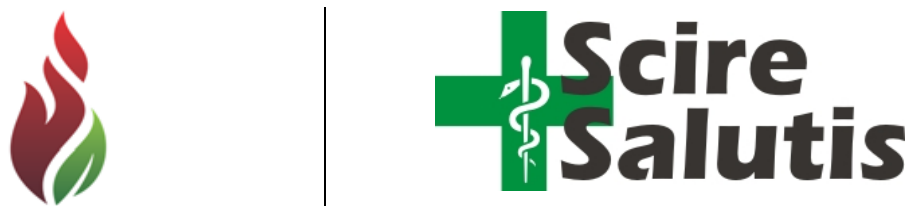

SUSTENERE

Publishing Corporation
Journals Homepage:

www.sustenere.co/journals

Scire Salutis, Aquidabã, v.4, n.1, Out, Nov, Dez 2013, Jan, Fev, Mar

2014.

\section{EFEITO DO LIAN GONG SHI BA FA NA CONDIÇÃO DOLOROSA DE TRABALHADORES DE UM SERVIÇO ADMINISTRATIVO}

\section{RESUMO}

As condições dolorosas relacionadas ao trabalho são frequentes e atingem cerca de $70 \%$ dos trabalhadores. Atividade física regular pode ajudar a reduzir o estresse e melhorar as condições para o sistema musculoesquelético. Programas de exercícios terapêuticos chineses são utilizados por milhares de pessoas, porém poucos estudos avaliaram seu efeito sobre trabalhadores de serviços administrativos em uma população ocidental. O objetivo do presente artigo foi avaliar o efeito do exercício terapêutico chinês Lian Gong Shi Ba Fa na condição dolorosa de trabalhadores de um serviço administrativo. Foi realizado um ensaio clínico analítico tendo como amostra 48 trabalhadores de um serviço administrativo. Foi utilizado o Questionário Nórdico de Sintomas Osteomusculares (QNMQ) e a escala visual analógica (EVA) associada à algometria de pressão. O programa de exercícios consiste em 18 exercícios realizados em 15 minutos, 2 vezes na semana e implementado em um período de 1 mês. O teste de Wilcoxon foi aplicado para verificar se houve alteração no EVA e na Algometria e correlacionar com a idade, sexo e tempo de serviço. Foi considerado significante $p<0,05$ para um intervalo de confiança de $95 \%$. Verificou-se uma diferença estatisticamente significante entre as medidas de EVA e da algometria em todas as análises. Observou-se que mulheres apresentaram melhores resultados com o programa. Com o presente estudo pôdese concluir que Lian Gong Shi $\mathrm{Ba} F a$ fornece bases para a intervenção em trabalhadores do serviço administrativo, o que contribui para o reconhecimento de técnicas resolutivas de tratamento da dor neste grupo específico de trabalhadores.

PALAVRAS-CHAVES: Dor; Atividade Física; Qi Gong.

\section{LIAN GONG SHI EFFECT OF BA FA IN THE PAINFUL CONDITION OF WORKERS OF AN ADMINISTRATIVE SERVICE}

\section{ABSTRACT}

The painful conditions related to work are common and affect about $70 \%$ of workers. Regular physical activity can help reduce stress and improve conditions for the musculoskeletal system. Chinese therapeutic exercise programs are used by thousands of people, but few studies have evaluated its effect on workers in administrative services in a Western population. The aim of this study was to evaluate the effect of Chinese Lian Gong Shi therapeutic exercise in Ba Fa painful condition of workers of an administrative department. An analytical clinical trial with a sample size of 48 workers an administrative service was held. The Nordic Musculoskeletal Questionnaire (QNMQ) and visual analogue scale (VAS) associated with algometry pressure was used. The exercise program consists of 18 exercises done in 15 minutes, 2 times a week and implemented in a period of 1 month. The Wilcoxon test was used to check changes in EVA and Algometry and correlate with age, sex and length of service. Was considered as $p<0.05$ for a confidence interval of $95 \%$. There was a statistically significant difference between the measures EVA and algometry in all analyzes. It was observed that women had better results with the program. With this study we concluded that Lian Gong Shi Ba Fa provides grounds for intervention in the administrative service workers, which contributes to the recognition of resolving technical treatment of pain in this particular group of workers.

KEYWORDS: Pain; Physical Activity; Qi Gong.

\section{ISSN 2236-9600}

\section{SECTION: Articles}

TOPIC: Fisioterapia

DOI: $10.6008 /$ SPC2236-9600.2014.001.0003

Kátia Nunes Sá

Universidade Tiradentes, Brasil http://lattes.cnpq.br/4313045041004715 katia.sa@gmail.com

Jessica Bahia Modesto

Universidade Tiradentes, Brasil http://lattes.cnpq.br/6341279682459024 jessicamodesto@gmail.com

Rafael dos Reis França

Universidade Tiradentes, Brasil http://lattes.cnpq.br/8925528623897587 francarafael@yahoo.com.br

Gilberto Antônio da Costa Júnior Universidade Tiradentes, Brasil http://lattes.cnpq.br/8700259928125329 gilbertoacj@gmail.com

Edmara Oliveira Mota

Universidade Tiradentes, Brasil http://lattes.cnpq.br/2902489621335730 mara1987om@hotmail.com

Mayanna Machado Freitas Universidade Tiradentes, Brasil http://lattes.cnpq.br/2088961229850444 mayannafreitas@hotmail.com

Received: 25/01/2014

Approved: 15/03/2014

Reviewed anonymously in the process of blind peer.

Referencing this:

SÁ, K. N.; MODESTO, J. B.; FRANÇA, R. R.; COSTA JÚNIOR, G. A.; MOTA, E. O.; FREITAS, M. M.. Efeito do Lian Gong Shi Ba Fa na condição dolorosa de trabalhadores de um serviço administrativo. Scire Salutis, Aquidabã, v.4, n.1, p.26-36, 2014. DOI: http://dx.doi.org/10.6008/SPC22369600.2014.001.0003 


\section{INTRODUÇÃO}

A dor musculoesquelética relacionada ao trabalho é um importante problema de saúde em muitos países industrializados (BRANDÃO et al., 2005; OSTERGREN et al., 2005; MAENO et al., 2006; ANDERSEN et al., 2007). Profissionais que atuam no serviço administrativo necessitam corresponder às demandas exigidas pela sua profissão. Para tanto, a jornada de trabalho envolve o manuseio repetitivo e por um longo período de tempo de recursos como computadores, papéis, calculadoras, dentre outros (MUROFUSE \& MARZIALI, 2001; ERRICO et al., 2007). A posição assumida por estes trabalhadores frente a estes recursos também é um ponto relevante que contribui para o aparecimento de distúrbios osteomusculares, juntamente com a super utilização das estruturas anatômicas do sistema osteomuscular e da falta de tempo de sua recuperação (MAENO et al., 2007; GONZALEZ et al., 2008).

As lesões podem se apresentar de diversas formas sendo caracterizadas pela ocorrência de vários sintomas concomitantes ou não, de aparecimento insidioso, geralmente nos membros superiores, tais como: dor, parestesia, sensação de peso e fadiga (MUROFUSE \& MARZIALI, 2001; ANDERSEN et al., 2007). As maiores incidências de distúrbios do sistema osteomioarticular ocorrem entre trabalhadores jovens, sendo as mulheres as mais acometidas (WALSH et al., 2004; ANDERSEN et al., 2008). Dados do INSS registram que $72 \%$ dos trabalhadores que recebem benefícios por incapacidade com síndrome cervicobraquial relacionada ao trabalho são bancários. Além disso, os benefícios por incapacidade concedidos a trabalhadores com tenossinovites e sinovites relacionadas ao trabalho, $55,3 \%$ eram concedidos a bancários que utilizavam sobremaneira os membros superiores por meio de microcomputadores (MAENO et al., 2006).

Mais de $50 \%$ dos profissionais que utilizam este recurso eletrônico relatam sintomas dolorosos em região de ombro, pescoço e braços (BOGERS et al., 2006). Dentre os trabalhadores brasileiros, 80 a $90 \%$ sofrem de doenças ocupacionais e $10,1 \%$ dos auxiliares administrativos do município de Salvador, apresentam alguma disfunção osteomuscular relacionada ao trabalho (YENG et al., 2001).

As causas de condições dolorosas são multifatoriais e têm influências na exposição biomecânica e psicossocial do indivíduo (ZHUANG, 2000; MUROFUSE \& MARZIALI, 2001). Fatores intrínsecos (como idade e sexo) e extrínsecos (como a profissão, carga horária, pausas para descanso, freqüência e intensidade e freqüência do trabalho) contribuem para o surgimento de disfunções osteomioarticulares (YENG et al., 2001; BONGERS et al., 2006).

Trabalhadores que executam suas tarefas em posturas estáticas ou que demandam posições de membros superiores elevados estão mais expostos a riscos de desenvolverem tais condições dolorosas e podem evoluir para quadros de dores crônicas causando estresse físico e emocional (BONGERS et al., 2006; ANDERSEN et al., 2007) A prática regular de atividade física tem sido reconhecida com um fator determinante para a prevenção e controle do estresse e de danos ao sistema osteomioarticular (OLIVEIRA, 2007; SANTOS et al., 2007). 
O Lian Gong Shi Ba Fa é uma ginástica terapêutica (Qi Gong) composta por três séries de dezoito exercícios específicos que priorizam ajustes globais. Ela foi criada em 1975 pelo médico chinês Dr. Zhuang Yuan Ming para tratar e prevenir síndromes doloridas no pescoço, nos ombros, na região lombar e nas pernas, bem como outros tipos de doenças crônicas, e que une o conhecimento médico ao movimento físico (ZHUANG, 2000; LIU \& HONG, 2005).

O Lian Gong tenta eliminar a sensação de dor restaurando o estado dos tecidos moles bem como promover a ativação de músculos e articulações de maneira simétrica e contínua. As características principais da sua execução são: movimento amplo, lento, contínuo, equilibrado e natural, coordenação espontânea da respiração com o movimento, exercícios abrangentes, simples e fáceis de aprender e executar, favorecendo a auto prevenção e a terapia (ZHUANG, 2000).

Incluir no período de trabalho destes profissionais administrativos uma ginástica terapêutica que possa ser aplicada de maneira contínua durante suas atividades diárias, pode ter um papel favorável no controle da dor musculoesquelética. Este estudo visou averiguar esta premissa na tentativa de certificar se há realmente benefícios na condição dolorosa de trabalhadores administrativos de um Hospital de referência do Estado da Bahia diante da implementação de um programa de exercícios terapêuticos chineses, Lian Gong Shi Ba Fa.

\section{MATERIAIS E MÉTODOS}

\section{Tipo de Estudo e Seleção da Amostra}

Este estudo consistiu em um ensaio clínico analítico com a amostra representada pelos funcionários do serviço administrativo do Hospital Geral do Estado (HGE), Salvador, Bahia. Como critérios de inclusão participaram do estudo funcionários ativos que realizavam tarefas de alta requisição manual (digitação e manuseio de documentos) e que apresentavam sintomas como dor ou desconforto em alguma região do corpo. Foram excluídos funcionários que não apresentavam condições dolorosas e que não aderiram ao programa com frequência mínima de $90 \%$.

Os trabalhadores voluntários assinaram o termo de livre consentimento de acordo com o decreto-lei $n^{\circ}$ 196/96 do Conselho Nacional de Saúde, após tomarem ciência dos objetivos e procedimentos do estudo.

\section{Coleta de Dados}

Os participantes primeiramente responderam a um questionário que levantou dados ocupacionais de carga horária de trabalho, tempo de serviço, intervalos de descanso, turno de trabalho, tipo de trabalho. Também responderam à versão brasileira do Questionário Nórdico de Sintomas Osteomusculares - QNMQ - para identificação de ocorrência de sintomas dolorosos 
nas diferentes regiões anatômicas, bem como o período de acometimento (PINHEIRO et al., 2002).

Outra ferramenta utilizada neste estudo foi a escala numérica de dor aplicada com a finalidade de mensurar a intensidade da dor na região considerada como a mais dolorosa pelo participante (POLETTO et al., 2004). A intensidade também foi avaliada através da algometria de pressão antes e após o programa de exercícios por meio do algômetro (MARCA) calibrado em $\mathrm{kgf} / \mathrm{cm} 2$. A mensuração foi realizada em um ângulo de noventa graus com a superfície corporal e com a taxa constante de aumento da pressão $(1 \mathrm{kgf} / \mathrm{cm} 2 / \mathrm{s})$ no ponto mais doloroso identificado pelo participante.

O Exercício terapêutico chinês Lian Gong Shi Ba Fa foi realizado durante um mês, com freqüência de duas sessões semanais no próprio horário e ambiente de trabalho. A série de exercícios foi aplicada por um período de quinze minutos. Uma semana antes de iniciar a pesquisa foram ministradas aulas com a finalidade de instruir os participantes sobre cada um dos dezoitos exercícios da série para compreensão da movimentação e ritmo adequados. Os exercícios de Liang Gong Shi Ba Fa foram realizados conforme o protocolo do Dr. Zhuang Yuan Ming (LIU, HONG, 2005). A Figura 1 demonstra passagens dos dezoito exercícios realizados no programa.

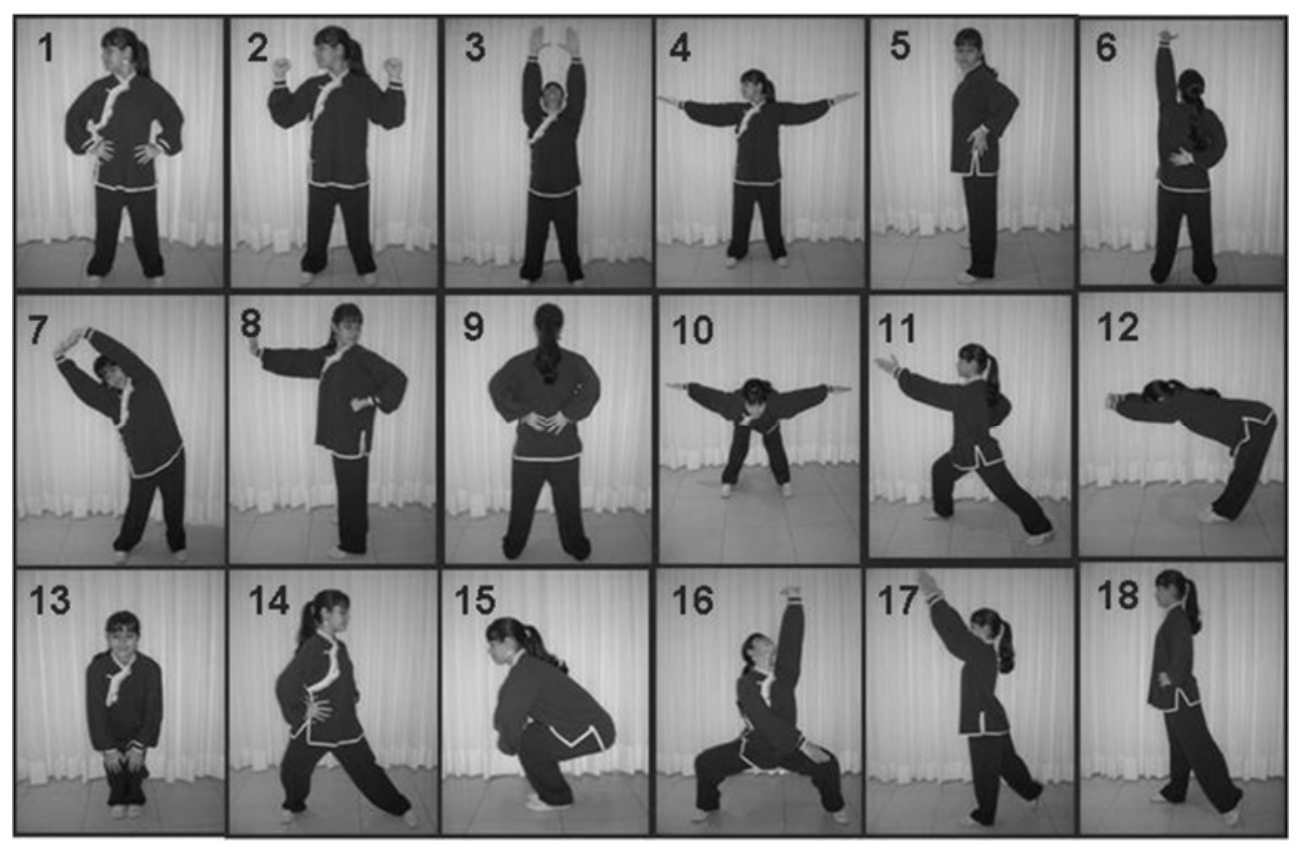

Figura 1: Sequência dos dezoito exercícios da primeira série do Lian Gong Shi Ba Fa. 1. Movimento do pescoço. 2. Arquear as mãos. 3. Estender as palmas para cima. 4. Expandir o peito. 5. Despregar as asas. 6. Levantar o braço de ferro. 7. Empurrar o céu e inclinar para o lado. 8. Girar a cintura e projetar as palmas. 9. Rodar a cintura com as mãos nos rins. 10. Abrir os braços e flexionar o tronco. 11. Espetar com a palma para o lado. 12. Tocar os pés com as mãos. 13. Rodar os joelhos. 14. Flexionar a perna e girar o tronco. 15. Flexionar e esticar as pernas. 16. Tocar o joelho e levantar e palma. 17. Abraçar o joelho contra o peito. 18.

Passos marciais.

Os participantes foram orientados a não praticarem os exercícios do Lian Gong fora dos horários delimitados durante o período da pesquisa, assim como a não realizar qualquer outra atividade física. 


\section{Análises Estatísticas}

A variável independente foi o programa de exercícios Lian Gong Shi Ba Fa e as variáveis dependentes foram os valores de EVA e a algometria de pressão. Como variáveis intervenientes, avaliou-se sexo, idade e tempo de serviço. Foi considerado significante $p<0,05$ para um intervalo de confiança de $95 \%$. As análises estatísticas foram realizadas através do teste de Wilcoxon por meio do software R 2.0.1.

\section{RESULTADOS}

Participaram do estudo quarenta e oito funcionários, trinta e nove eram do sexo feminino e nove participantes eram do sexo masculino, com idade entre vinte e três e cinquenta e oito anos (média $=42,45$ ). Dos participantes, 83,3\% relataram não realizar atividade física regular, 54,4\% possuem tempo serviço entre quinze e vinte e cinco anos e $79,1 \%$ possuem carga horária de quarenta horas semanais. As características da população de estudo estão delimitadas na Tabela 1.

Tabela 1: Características da população de estudo ( $\mathrm{N}=48)$.

\begin{tabular}{lcc}
\hline Variáveis & $\mathbf{N}=\mathbf{4 8}$ & $\%$ \\
\hline $\begin{array}{l}\text { Sexo } \\
\text { Masculino }\end{array}$ & 9 & 18,8 \\
Feminino & 39 & 81,2 \\
& & \\
Idade (anos) & 23 & 47,9 \\
$\mathbf{2 3}-\mathbf{4 0}$ & 25 & 52,1 \\
$\mathbf{4 1 - 5 7}$ & & \\
Tempo de serviço (anos) & 22 & 45,8 \\
$\mathbf{1}-\mathbf{1 4}$ & 26 & 54,2 \\
$\mathbf{1 5}-\mathbf{2 5}$ & & \\
Carga horária semanal & 9 & 18,8 \\
$\mathbf{3 0}$ & 38 & 79,1 \\
$\mathbf{4 0}$ & 1 & 2,1 \\
$\mathbf{6 0}$ & & \\
Atividade física & 8 & 16,7 \\
$\quad$ Sim & 40 & 83,3 \\
$\quad$ Não & &
\end{tabular}

Foi encontrada diferença estatisticamente significante em todas as comparações realizadas $(p<0,05)$. Com relação à intensidade da dor medida pela EVA, verificou-se que o sexo feminino obteve menores valores após o programa comparado ao sexo masculino, embora ambos tenham apresentado resultados significativos. Além disso, pode-se constatar que não houve diferenças com relação à faixa etária e o tempo de serviço, demonstrando resultados positivos para todos os estratos (Tabela 2). 
Tabela 2: Média e desvio-padrão de EVA antes e depois, segundo o sexo, a idade e o tempo de serviço.

\begin{tabular}{|c|c|c|c|c|c|}
\hline \multirow[t]{2}{*}{ Variáveis } & \multicolumn{4}{|l|}{$\begin{array}{l}\text { EVA } \\
\text { Antes }\end{array}$} & \multirow{2}{*}{$\begin{array}{l}\text { P-valor } \\
\text { (Teste de Wilcoxon) }\end{array}$} \\
\hline & Média & DP & Média & DP & \\
\hline \multicolumn{6}{|l|}{ Sexo } \\
\hline Masculino & 2,89 & 2,53 & 2,00 & 2,00 & 0,0231 \\
\hline Feminino & 5,46 & 2,74 & 4,38 & 2,50 & 0,0000 \\
\hline \multicolumn{6}{|l|}{ Idade (anos) } \\
\hline $23-40$ & 5,09 & 3,07 & 3,87 & 2,80 & 0,0002 \\
\hline $41-57$ & 4,88 & 2,71 & 4,00 & 2,40 & 0,0006 \\
\hline \multicolumn{6}{|c|}{ Tempo de serviço (anos) } \\
\hline $1-14$ & 4,55 & 3,04 & 3,45 & 2,76 & 0,0005 \\
\hline $15-25$ & 5,35 & 2,71 & 4,35 & 2,38 & 0,0002 \\
\hline
\end{tabular}

DP = Desvio-padrão.

Estabelecendo-se correlação entre as variáveis intervenientes e a algometria de pressão, podemos destacar que o grupo feminino obteve melhores valores comparados ao grupo masculino. Com relação à idade e tempo de serviço não houve diferença significante entre as categorias (Tabela 3), apontando para o efeito positivo em todos os segmentos estudados.

Tabela 3: Média e desvio-padrão da algometria antes e depois, segundo o sexo, a idade e o tempo de serviço.

\begin{tabular}{|c|c|c|c|c|c|}
\hline \multirow[t]{2}{*}{ Variáveis } & \multicolumn{2}{|c|}{$\begin{array}{l}\text { Algometria } \\
\text { Antes }\end{array}$} & \multicolumn{2}{|l|}{ Depois } & \multirow{2}{*}{$\begin{array}{l}\text { P-valor } \\
\text { (Teste de Wilcoxon) }\end{array}$} \\
\hline & Média & DP & Média & DP & \\
\hline \multicolumn{6}{|l|}{ Sexo } \\
\hline Masculino & 5,86 & 2,04 & 6,20 & 2,11 & 0,0115 \\
\hline Feminino & 4,36 & 1,72 & 4,73 & 1,74 & 0,0000 \\
\hline \multicolumn{6}{|l|}{ Idade (anos) } \\
\hline $23-40$ & 4,57 & 2,03 & 4,97 & 2,06 & 0,0005 \\
\hline $41-57$ & 4,71 & 1,73 & 5,04 & 1,75 & 0,0008 \\
\hline \multicolumn{6}{|c|}{ Tempo de serviço (anos) } \\
\hline $1-14$ & 4,80 & 1,98 & 5,17 & 2,03 & 0,0008 \\
\hline $15-25$ & 4,51 & 1,78 & 4,86 & 1,78 & 0,0005 \\
\hline
\end{tabular}

Quanto aos sintomas osteomusculares presentes (Tabela 4) nos últimos doze meses, observou-se que as regiões mais acometidas foram pescoço/cervical, ombro, região dorsal e lombar. A região que mais sofre constantemente algum problema foi região de pescoço/cervical.

Tabela 4: Frequência de problemas (dor, desconforto ou dormência), nos últimos 12 meses, de acordo com a região $(\mathrm{N}=48)$.

\begin{tabular}{|c|c|c|c|c|}
\hline Regiões & $\begin{array}{l}\text { Não } \\
\text { n (\%) }\end{array}$ & $\begin{array}{l}\text { Raramente } \\
\mathrm{n}(\%)\end{array}$ & $\begin{array}{l}\text { Com freqüência } \\
\text { n (\%) }\end{array}$ & $\begin{array}{l}\text { Sempre } \\
\text { n (\%) }\end{array}$ \\
\hline $\begin{array}{l}\text { Pescoçol } \\
\text { Cervical }\end{array}$ & $2(4,2)$ & $15(31,3)$ & $18(37,4)$ & $13(27,1)$ \\
\hline Ombro & $7(14,6)$ & $13(27,1)$ & $19(39,5)$ & $9(18,8)$ \\
\hline Braços & $17(35,4)$ & $13(27,1)$ & $13(27,1)$ & $5(10,4)$ \\
\hline Cotovelos & $30(62,5)$ & $10(20,8)$ & $6(12,5)$ & $2(4,2)$ \\
\hline Antebraço & $37(77,1)$ & $4(8,3)$ & $4(8,3)$ & $3(6,3)$ \\
\hline Punhos/Mãos/Dedos & $34(70,9)$ & $5(10,4)$ & $4(8,3)$ & $5(10,4)$ \\
\hline Região Dorsal & $11(22,9)$ & $9(18,8)$ & $23(47,9)$ & $5(10,4)$ \\
\hline Região lombar & $7(14,6)$ & $19(39,5)$ & $15(31,3)$ & $7(14,6)$ \\
\hline Quadril/Membros inferiores & $15(31,3)$ & $17(35,3)$ & $7(14,6)$ & $9(18,8)$ \\
\hline
\end{tabular}

$\mathrm{n}=$ número de pacientes. 
As regiões mais acometidas nos últimos sete dias precedentes à data da avaliação foram: região de pescoço/cervical, ombros, região dorsal e região lombar. A região referida por $23 \%$ dos participantes como a constantemente atingida por problemas foi a região de pescoço/cervical. (Tabela 5).

Tabela 5: Frequência de problemas (dor, desconforto ou dormência), nos últimos 7 dias, de acordo com a região $(\mathrm{N}=48)$.

\begin{tabular}{|c|c|c|c|c|}
\hline Regiões & $\begin{array}{l}\text { Não } \\
\text { n (\%) }\end{array}$ & $\begin{array}{l}\text { Raramente } \\
\text { n (\%) }\end{array}$ & $\begin{array}{l}\text { Com freqüência } \\
\text { n (\%) }\end{array}$ & $\begin{array}{l}\text { Sempre } \\
\text { n (\%) }\end{array}$ \\
\hline Pescoço/Cervical & $5(10,4)$ & $16(33,3)$ & $16(33,3)$ & $11(23,0)$ \\
\hline Ombro & $16(33,3)$ & $9(18,8)$ & $13(27,1)$ & $10(20,8)$ \\
\hline Braços & $29(60,4)$ & $7(14,6)$ & $7(14,6)$ & $5(10,4)$ \\
\hline Cotovelos & $41(85,3)$ & $2(4,2)$ & $2(4,2)$ & $3(6,3)$ \\
\hline Antebraço & $41(85,4)$ & $0(0,0)$ & $4(8,3)$ & $3(6,3)$ \\
\hline Punhos/Mãos/Dedos & $37(77,1)$ & $4(8,3)$ & $2(4,2)$ & $5(10,4)$ \\
\hline Região Dorsal & $17(35,4)$ & $8(16,7)$ & $18(37,5)$ & $5(10,4)$ \\
\hline Região lombar & $14(29,1)$ & $13(27,1)$ & $13(27,1)$ & $8(16,7)$ \\
\hline Quadril/Membros inferiores & $25(52,0)$ & $7(14,6)$ & $7(14,6)$ & $9(18,8)$ \\
\hline
\end{tabular}

$\mathrm{n}=$ número de participantes

A Tabela 6 demonstra as regiões que contribuem para o absenteísmo em profissionais do setor administrativo. Verificou-se que a região mais relatada foi a região de pescoço/cervical, seguida pela região de ombros.

Tabela 6: Frequência de não realização das atividades (dor, desconforto ou dormência), nos últimos 12 meses, de acordo com a região $(\mathrm{N}=48)$.

\begin{tabular}{|c|c|c|}
\hline Regiões & $\begin{array}{l}\text { Não } \\
\text { n (\%) }\end{array}$ & $\begin{array}{l}\text { Sim } \\
\text { n (\%) }\end{array}$ \\
\hline Pescoço/Cervical & $27(56,2)$ & $21(43,8)$ \\
\hline Ombro & $33(68,7)$ & $15(31,3)$ \\
\hline Braços & $45(93,7)$ & $3(6,3)$ \\
\hline Cotovelos & $46(95,8)$ & $2(4,2)$ \\
\hline Antebraço & $46(95,8)$ & $2(4,2)$ \\
\hline Punhos/Mãos/Dedos & $43(89,6)$ & $5(10,4)$ \\
\hline Região Dorsal & $46(95,8)$ & $2(4,2)$ \\
\hline Região lombar & $40(83,3)$ & $8(16,7)$ \\
\hline Quadril/Membros inferiores & $42(87,5)$ & $6(12,5)$ \\
\hline
\end{tabular}
$\mathrm{n}=$ número de pacientes

\section{DISCUSSÃO}

O presente estudo teve como objetivo verificar o efeito do exercício terapêutico chinês Lian Gong Shi Ba Fa na condição dolorosa de trabalhadores de um serviço administrativo. Observouse melhora significativa da dor neste grupo específico de profissionais com a implementação do programa de exercícios criado pelo Dr. Zhuang.

Dos 48 trabalhadores selecionados, 83,3\% relataram que não realizavam atividade física regular. Alguns autores demonstram que a própria natureza do trabalho ao proporcionar meios de conforto e facilidade para a execução das tarefas favorece um estilo de vida sedentário (SILVA \& JUVENCIO, 2004; SANTOS et al., 2007). Além disso, fatores tais como: carga de trabalho elevada, reduzido deslocamento do posto de trabalho, aspectos psicossociais e ergonômicos dentro do ambiente profissional contribuem para a hipocinesia evidenciada entre a classe trabalhadora, pode facilitar esta condição (SILVA \& JUVENCIO, 2004; MACIEL, 2007). 
Foi verificada uma redução na intensidade da dor com a utilização do programa de exercícios terapêuticos chineses em todas as análises realizadas, demonstrando ser esta técnica um recurso simples, de baixo custo e efetivos resultados para o controle da condição dolorosa em curto prazo. Alguns autores defendem que exercícios específicos destinados ao ambiente de trabalho podem prevenir doenças ocupacionais, promover o alívio das dores corporais, reduzir os casos de LER/DORT e estabelecer um aumento da produtividade e um maior retorno financeiro das empresas (NURMINEN et al., 2002; OLIVEIRA, 2007). Um estudo realizado em 2004 enfatiza que trabalhadores praticantes de atividade física regular por no mínimo dez meses reduzem o risco de aparecimento de sintomas na região de pescoço e ombro e de absenteísmo (VAN DEN HEUVEL et al., 2007). Além disso, foi evidenciado que o grupo constituído por trabalhadores do sexo feminino apresentou melhores resultados que o grupo masculino. Este fato se relaciona com achados de autores que demonstram ganhos significativos da atividade física em mulheres, no ambiente de trabalho. Pesquisas confirmaram uma melhora na habilidade necessária para desenvolver o trabalho bem como na demanda psicológica de mulheres trabalhadoras (NURMINEN et al., 2002). Este achado complementa a premissa de autores que utilizaram um programa de exercícios em mulheres trabalhadoras portadoras de dor crônica em região do pescoço com intensidade de no mínimo cinco na escala visual analógica. Estes autores verificaram redução do quadro álgico após treinamento da musculatura envolvida (ANDERSEN et al., 2008).

Com relação às regiões mais acometidas por este grupo específico de trabalhadores, o estudo evidencia que há predomínio de sintomas dolorosos nas regiões de pescoço/cervical e de ombros. A literatura confirma a crescente demanda destas regiões para manipular eletrônicos como computadores por longos períodos através da jornada de trabalho e contribui para o aparecimento de sintomas e queixas álgicas (SHANAHAN \& JEZUKAITIS, 2006; ELTAYEB et al., 2007). Em pesquisa desenvolvida em 2004, identificou-se que dos duzentos e sessenta e quatro trabalhadores de uma empresa nacional da Holanda, $54 \%$ dos digitadores relataram pelo menos uma queixa nestas regiões no período de um ano. A alta prevalência de dor foi pontuada nas regiões de ombro e pescoço com 33\% e 31\% respectivamente, seguida de queixas em mão e braços (11 e 12\%) (ELTAYEB et al., 2007). Outro estudo epidemiológico foi realizado com o intuito de investigar as interações potenciais entre riscos físicos e psicossociais no local de trabalho que podem estar associados com disfunções musculoesqueléticas em região de pescoço e cintura escapular delimitando achados relevantes. Dos trabalhadores, 55\% referiram sentir algum desconforto ou dor na região de extremidade superior e cintura escapular. Foi constatado pelos autores que a associação entre fatores físicos e psicossociais aumentam o risco de desenvolvimento de desordens na cintura escapular e membros superiores (DEVEREUX et al., 2002).

A melhora significativa destes profissionais com a utilização dos exercícios terapêuticos chineses Lian Gong Shi Ba Fa pode ser evidenciada através das características peculiares desta 
prática. Segundo o Dr. Zhuang este grupo de exercícios promovem melhoras na circulação do sangue equilibram a função dos nervos e fortalece a constituição física do indivíduo (ZHUANG, 2000).

Além disso, como a região mais acometida pelos trabalhadores administrativos foram as regiões de membros superiores e região escapular, a série de exercícios escolhida foi a voltada para a prevenção e tratamento de dores nestas regiões. Estes exercícios promovem alongamento, fortalecimento e relaxamento destas estruturas de maneira global e sequenciada através de um ritmo e respiração específica.

Uma pesquisa randômica e prospectiva foi realizada, em 2007, com o objetivo de comparar a eficácia do Qi Gong (exercício terapêutico chinês) médico com outros tipos de exercícios terapêuticos em pacientes com dor prolongada no pescoço. Homens e mulheres com dor não específica no pescoço por mais de três meses foram incluídos no estudo. Os autores demonstraram que não houve diferenças significativas entre ambas terapêuticas, porém as duas intervenções diminuem a dor no pescoço e o grau de disfunção e o efeito pode ser mantido por um ano. O Qi Gong inicia a "resposta de relaxamento" que é fomentada pela concentração, libera a mente das distrações, promove a dilatação de vasos capilares sanguíneos e a nutrição tecidual (JOUPER et al., 2006; LASINGER et al., 2007).

\section{CONCLUSÕES}

Pode-se concluir com o presente estudo que os dezoito primeiros exercícios do programa Lian Gong Shi Ba Fa foram eficazes na melhora da condição dolorosa de trabalhadores de um setor administrativo. Como se trata de um programa de baixo custo e com um efeito tão positivo em curto período de tempo, sugere-se que sejam aplicados em grupos maiores e no modelo de ensaio clínico randomizado para que as inferências possam ser aplicáveis a um grupo maior de trabalhadores que sofrem de dor musculoesquelética.

\section{REFERÊNCIAS}

ANDERSEN J.H.; HAARH J. P.; FROST P.. Risk Factors for More Severe Regional Musculoskeletal Symptoms. Arthritis \& Rheumatism, v.56, n.4, 2007.

ANDERSEN, L.L.; KJAER, M.; ANDERSEN, C.H.; HANSEN, P.B.; ZEBIS, M.K.; HANSEN, K.; SJOGAARD, G.. Muscle Activation During Selected Strength Exercises in Women With Chronic Neck Muscle Pain. Physical Therapy, v.88, n.6, p.703-711, 2008.

BERNAARDS, C. M.; ARIËNS, G. A.; HILDEBRANDT, V. H.. The (cost-) effectiveness of a lifestyle physical activity intervention in addition to a work style intervention on the recovery from neck and upper limb symptoms in computer workers. BMC Musculoskeletal Disorders, v.7, n.80, 2006.

BRANDÃO, A. G.; HORTA, B. L.; TOMASI, E.. Sintomas de distúrbios osteomusculares em bancários de Pelotas e região: prevalência e fatores associados. Revista Brasileira de Epidemiologia, v.8, n.3, 2005. 
BONGERS, P. M.; IJMKER, S.; HEUVEL, S.. Epidemiology of work related neck and upper limb problems: Psychosocial and personal risk factors (Part I) and effective interventions from a bio behavioural perspective (Part II). Journal of Ocupacional Rehabilitation, v.16, n.3, p.279-302, 2006.

DEVEREUX, J. J.; VLACHONIKOLIS, I. G.; BUCKLE, P. W.. Epidemiological study to investigate potential interaction between physical and psychosocial factors at work that may increase the risk of symptoms of musculoskeletal disorder of the neck and upper limb. Occupation Environmental Medicine, v.59, p.15-28, 2002.

ELTAYEB, S.; STAAL, J. B.; KEENES, J.; LAMBERTS, P. H. G.; DE BIE, R. A. Prevalence of complaints of arm, neck and shoulder among computer office workers and psychometric evaluation of a risk factor questionnaire. BMC Musculoskeletal Disorders, v.8, n.68, p.100-120, 2007.

ERRICO, A. D.; GORE, R.; GOLD, J. E.; PARK, J.S.; PUNNETT, L.. Medium- and long-term reproducibility of self-reported exposure to physical ergonomics factors at work. Applied Ergonomics, v.38, n.2, p.167175, 2007.

GONZALEZ, L. R.; LUCCA, S. R.; KITAMURA, S.. Contribuições para investigação de lesões por esforços repetitivos - distúrbios osteomusculares relacionados com o trabalho em membros superiores. Revista Brasileira Cliníca Medica, v.6, n.2, 2008.

JOUPER, J.; HASSMEN, P.; JOHANSSON, M.. Qi Gong Exercise with concentration predicts increased healthy. American Journal of Chinese Medicine, v.34, n.6, p.949-957, 2006.

LASINGER, B.; LARSSON, E.; PERSSON, L.; CARLSSON, J. Y.. Qigong and exercise therapy in patients withlong-term neck pain. SPINE, v.32, n.22, p.2415-1422, 2007.

LIU, G. W.; HONG, J. P.. Tratado contemporâneo de acupuntura e moxabustão. São Paulo: CEIMEC, 2005.

MACIEL, M. G.. Análise da relação entre o estilo de vida e a percepção subjetiva da fadiga em trabalhadores para a implantação da ginástica laboral. Cinergis, v.8, n.1, p.16-24, 2007.

MAENO M.; SALERNO V.; ROSSI, D.A.G.; FULLER, R.. Protocolos de atenção integral à Saúde do Trabalhador de Complexidade Diferenciada. Brasília: Ministério da Saúde, 2006.

MARTINS, C. O.; DUARTE, M. F.. Efeitos da ginástica laboral em servidores da Reitoria da UFSC. Revista Brasileira Ciência e Movimento, v.8, p.12-26, n.4, 2000.

MUROFUSE N.; MARZIALE, M.. Mudanças no trabalho e na vida de bancários portadores de lesões por esforços repetitivos: LER. Revista Latino Americana de Enfermagem, v.9, n.4, 2001.

NURMINEN, E.; MALMIVAARA, A.; ILMARINEN, J.; YLOSTALO, P.; MUTANEN, P.; AHONEN, G.; ARTO, T.. Effectiveness of a worksite exercise program with respect to perceived work ability and sick leaves among women with physical work. Scand Journal Work Environ Health, v.28, n.2, p.85-93, 2002.

OSTERGREN P. O.; HANSON B. S.; BALOGH I.; EKTOR-ANDERSEN, J.; ISACSSON, S.O.; ORBAEK, P.; WINKEL, J.; ISACSSON, S. O.. Incidence of Shoulder and Neck Pain in a Working Population: Effect Modification Between Mechanical and Psychosocial Exposures at Work? Results from a One Year Follow up of the Malmo Shoulder and Neck Study Cohort. Journal of Epidemiology Community Health, v.59, n.9, p.721-728, 2005.

OLIVEIRA, J. R.. A importância da ginástica laboral na prevenção de doenças ocupacionais. Revista de Educação Física, v.3, n.139, p.52-61, 2007.

PINHEIRO, F.; TROCCOLI, B. T.; CARVALHO, C. V.. Validação do Questionário Nórdico de Sintomas Osteomusculares como medida de morbidade. Revista de Saúde Pública, v.36, n.3, p.307-312, 2002.

POLETTO P. R.; COURY G.; HELENICE, J.C.G.; WALSH, I.A.P.; MATTIELLO, S. M.. Correlação entre métodos de auto-relato e testes provocativos de avaliação da dor em indivíduos portadores de distúrbios osteomusculares relacionados ao trabalho. Revista Brasileira de Fisioterapia, v.8, n.3, p.223-229, 2004. 
ROBROEK, S. J.; LENTHE, F. J.; EMPELEN, E. V.; BURDORF, A. Determinants of participation in worksite health promotion programmes: a systematic review. International Journal of Behavioral Nutrition and Physical Activity, n.6, v.26, 2009.

SANTOS, A.F.; ODA, J.Y.; NUNES, A. P.; GONÇALVES, L.; GARNÉS, F. L. S.. Benefícios da ginástica laboral na prevenção dos distúrbios osteomusculares relacionados ao trabalho. Arquivo de Ciência de Saúde Unipar, v.11, n.2, p.99-106, 2007.

SHANAHAN, E.M.; JEZUKAITIS, P.. Work related upper limb disorders. Australian Family Physician, v.35, n.12, 2006.

SILVA C.D.; JUVÊNCIO J. F.. Diagnóstico da aptidão física relacionada à saúde em trabalhadores de escritório da universidade federal de viçosa. Revista Brasileira de Cineantropometria \& Desempenho Humano, v.6, n.1, 2004.

VAN DEN HEUVEL, S. G.; HEINRICH, J.; JANS, M. S.; VAN DER BEEK, A. J.; BONGERS, P. M.. The effect of physical activity in leisure time on neck and upper limb symptoms. Applied Ergonomics, v.38, n.1, p.260-267, 2007.

YENG L.T.; JACOBSEN T. M.; APARECIDA R. M.. Distúrbios Osteomusculares Relacionado ao Trabalho. Revista MED, v.80, n.2, 2001.

WALSH, I.; CORRAL, S.; FRANCO, R. N.; CANETTI, E. E. F.; ALEM, M. E. R.; COURY, H. J. C. G.. Capacidade para o trabalho em indivíduos com lesões músculo-esqueléticas crônicas. Revista de Saúde Pública, v.38, n.3, p.149-156, 2004.

ZHUANG, Y. M.. Lian Gong Shi Ba FA ginástica terapêutica e preventiva. São Paulo: Pensamento, 2000. 